\title{
Mechanistic Study of the Collision-Induced Dissociation of Sodium-Cationized Polylactide Oligomers: A Joint Experimental and Theoretical Investigation
}

\author{
Julien De Winter, ${ }^{\text {a }}$ Vincent Lemaur, ${ }^{\mathrm{b}}$ Philippe Marsal, ${ }^{\mathrm{b}, \mathrm{d}}$ \\ Olivier Coulembier, ${ }^{\mathrm{c}}$ Jérôme Cornil, ${ }^{\mathrm{b}}$ Philippe Dubois, ${ }^{\mathrm{c}}$ and \\ Pascal Gerbaux ${ }^{\mathrm{a}}$ \\ ${ }^{a}$ Mass Spectrometry Research Group, Centre Interdisciplinaire de Spectrométrie de Masse (CISMa), \\ University of Mons, Mons, Belgium \\ ${ }^{\mathrm{b}}$ Laboratory for Chemistry of Novel Materials, University of Mons, Mons, Belgium \\ ${ }^{c}$ Center of Innovation and Research in Materials and Polymers (CIRMAP), Laboratory of Polymeric and \\ Composite Materials, University of Mons, Mons, Belgium \\ ${ }^{\mathrm{d}}$ CINaM-CNRS, Université Aix-Marseille 2 and 3, Campus de Luminy, Marseille, France
}

The low-kinetic energy collision-induced dissociation (CID) behavior of different sodiumcationized polylactide (PLA) oligomers was thoroughly investigated to shed some light on the analytical potentialities of CID experiments in the context of polymer characterization. Indeed, investigation of several end-groups modified PLA reveals that, in addition to the expected end-group specific dissociations, collisionally-excited PLA.Na ${ }^{+}$suffer from a backbone cleavage. The so-obtained sodium-bound dimer cations consecutively undergo the loss of a monomeric residue that corresponds to neutral acrylic acid. The experimental observations, performed on a hybrid Q-ToF instrument, were totally corroborated by a theoretical study involving DFT calculations, molecular mechanics, and molecular dynamics calculations. (J Am Soc Mass Spectrom 2010, 21, 1159-1168) (c) 2010 American Society for Mass Spectrometry

$\mathrm{A}$ $\mathrm{t}$ the present time, intensive researches are devoted to the preparation of biodegradable and biocompatible polymers. This field of research represents an up-growing domain since their applications are as diverse as their possible structures. For instance, biodegradable and biocompatible polymers have been used as biomaterials for various applications such as implant components [1] and bone cements [2]. Such biodegradable polymers are often designed to degrade hydrolytically so that most of the widely studied biodegradable polymers belong to the polyester family and include polylactide (PLA), polyglycolide (PG), and associated copolymers [3]. PLA is certainly one of the most important synthetic biodegradable and biocompatible polymers attracting a huge interest for new and versatile synthetic procedures [4]. Polylactide and poly(lactic acid) are, respectively, obtained by ring-opening polymerization (ROP) of lactide, the cyclic dimer of lactic acid, or by polycondensation of lactic acid.

In the course of our research on the polymerization of lactide using non-organometallic catalysts such as $N$-heterocyclic carbenes, superbases, fluorinated tertiary alcohols, thiourea derivatives, and phosphazenes

Address reprint requests to Dr. P. Gerbaux, Mass Spectrometry Research Group, Centre Interdisciplinaire de spectrométrie de masse, University of Mons, 20, Place du Parc, 7000 Mons, Belgium. E-mail: Pascal.Gerbaux@ umons.ac.be
[5-7], we decided to explore the potential of mass spectrometry to provide an in-depth characterization of synthetic polymers in addition to well-established methodologies, such as gel permeation chromatography (GPC) and nuclear magnetic resonance (NMR). Electrospray ionization (ESI) and matrix-assisted laser desorption ionization (MALDI) represent the most significant recent developments in ionization techniques for mass spectrometry analysis of synthetic polymers [8]. State-of-the-art mass spectrometry tools hold the promise of providing not only absolute molecular mass distributions [9] but also qualitative end-group and repeat-unit composition [10], as well as structural information such as branching and intramolecular ring formation [11]. By definition, MS experiments provide the masses of the individual oligomers prepared during the polymer synthesis. On the basis of the $\mathrm{m} / \mathrm{z}$ ratio of the detected cationized oligomers, compositional pieces of information such as the number and type of monomer units but also the nature of the incorporated end-groups can be derived. Nowadays, tandem mass spectrometry (MSMS) is emerging as an essential analytical methodology when accurate structural characterization of polymers cannot be unambiguously derived from single-stage MS experiments [12]. Recent MSMS investigations were successfully used to characterize individual functional groups of new polymers, to make 
a clear-cut distinction between isomeric oligomers, and even to determine copolymer sequences [13].

Amongst the tandem mass spectrometry tools, collisioninduced dissociation (CID) methodology represents the privileged technique for a deep structural characterization of gas-phase ions [14, 15]. CID experiments rely on the gas-phase collisional activation of a specific massselected ion. Given the non-elastic nature of such collisional events, the ions suffer from an internal energy increase that ultimately induces bond dissociations. The recording of the fragment ions affords the so-called CID spectrum that can prove helpful in the context of structural characterization. Reconstruction of the ion primary structure on the basis of the detected fragments is often not straightforward and requires the knowledge of the ion fragmentation mechanism. An established decomposition mechanism yields a template to use the fragment ions to reconstruct the connectivity of any related molecules. In the context of polymer analysis, recent papers describe the use of CID methodology for polymer ion characterization. Indeed, the CID behavior of different types of polymers, such as polyisobutylene [16], polystyrene [17], polymethylmethacrylate [18], polyether [19], and polyester [10-13] were investigated.

In direct connection with the scope of our work, two recent papers have reported the CID characterization of sodium-cationized PLA oligomers[13, 20]. Both papers describe that the dissociations of collisionally-excited cations are always initiated by a hydrogen atom migration through a favorable six-membered ring transitionstate ultimately leading to backbone cleavages at the ester binding sites. It was inferred that such dissociations can occur at both sides of the oligomer chains.

To further investigate these dissociation reactions and reach a more detailed mechanistic aspect, we have performed a joint experimental and theoretical study of the CID behavior of several sodium-cationized PLA oligomers prepared by electrospray ionization.

\section{Experimental}

\section{Materials}

All the samples are commercially available except polylactic acid (theoretical $\mathrm{Mn}=1000$ ) received from Galactic S.A. (Escanaffles, Belgium), whereas $\alpha$-methyl, $\omega$ hydroxylpolylactide and $\alpha$-methyl, $\omega$-acetatepolylactide were prepared following the procedures described here under. L-lactide monomer is first dried by successive azeotropic distillations of toluene and then stored in glove box.

\section{Syntheses}

\section{$\alpha$-Methyl, $\omega$-Hydroxylpolylactide}

In a glove box, $1.06 \mathrm{~g}\left(\mathrm{nLA}=7.3510^{-3} \mathrm{~mol}\right)$ of dried L-lactide was introduced in a previously flamed and nitrogen purged two-neck flask equipped with a three- way stopcock and a magnetic stirring bar. Outside of the box, $0.215 \mathrm{~g}$ of 5-methoxy-1,3,4-triphenyl-4,5dihydro- $1 \mathrm{H}-1,2,4$-triazole (triazole, $n=6,510^{-4} \mathrm{~mol}$ ) was added to the L-lactide under nitrogen atmosphere. The triazole/L-lactide mixture was then charged by 3.5 $\mathrm{mL}$ of dried toluene under nitrogen by using previously flame-dried syringes. The mixture was then heated at $90{ }^{\circ} \mathrm{C}$ under stirring. After $5 \mathrm{~h}$, the polymerization was stopped by adding a few drops of carbon disulfide. The obtained polymer was then precipitated out from cold heptane, filtered and dried under vacuum at $40{ }^{\circ} \mathrm{C}$.

${ }^{1} \mathrm{H}-\mathrm{NMR}\left(300 \mathrm{MHz}, \mathrm{CDCl}_{3}, \delta(\mathrm{ppm})\right)$ : 1.2-1.5 (d, $3(2 \mathrm{n}) \mathrm{H}), 3.7(\mathrm{~m}, 3 \mathrm{H}), 4.4(\mathrm{q}, 1 \mathrm{H} ; \mathrm{CH}-\mathrm{OH}), 5-5.3$ (q, $(2 \mathrm{n}-1) \mathrm{H})$.

\section{$\alpha$-Methyl, $\omega$-Acetatepolylactide}

The as-obtained $\alpha$-methyl, $\omega$-hydroxylpolylactide $(\mathrm{Mn}=$ $1200 \mathrm{~g} . \mathrm{mol}^{-1} ; 100 \mathrm{mg}$ ) and $41 \mathrm{mg}$ of dimethyl-aminopyridine (DMAP) were introduced without any particular caution in a one neck flask. $8 \mathrm{ml}$ of toluene were introduced to dissolve the reactives. When the solution was at reflux, $24 \mu \mathrm{L}$ of acetyl chloride were introduced. After $20 \mathrm{~h}$ of reaction, the solution was directly filtered before precipitation in cold methanol.

${ }^{1} \mathrm{H}-\mathrm{NMR}\left(300 \mathrm{MHz}, \mathrm{CDCl}_{3}, \delta(\mathrm{ppm})\right)$ : 1.2-1.5 (d, $3(2 n+1) H), 3.7(m, 3 H), 2.05(s, 3 H), 5-5.3(q, 2 n H)$.

\section{Mass Spectrometry Investigations}

All experiments were performed on a Waters (Manchester, UK) QToF2 mass spectrometer. The polymer solutions $\left(1 \mathrm{mg} \cdot \mathrm{mL}^{-1}\right.$ in acetonitrile $/ 1 \mathrm{mg} \cdot \mathrm{mL}^{-1} \mathrm{NaI}$ in acetonitrile: 9/1) were delivered to the ESI source by a Harvard Apparatus syringe pump at a flow rate of $5 \mu \mathrm{L} / \mathrm{min}$. Typical ESI conditions were: capillary voltage, $3.1 \mathrm{kV}$; cone voltage, $30 \mathrm{~V}$; source temperature, $80^{\circ} \mathrm{C}$; desolvation temperature, $120^{\circ} \mathrm{C}$. Dry nitrogen was used as the ESI gas. These ionization conditions led to the formation of abundant [PLA $+\mathrm{Na}^{+}$] cations for each polymer under investigation. The quadrupole was set to pass ions from 100 to $3000 \mathrm{Th}$ and all ions were transmitted into the pusher region of the time-of-flight analyzer for mass-analysis with $1 \mathrm{~s}$ integration time. Data were acquired in continuum mode until acceptable average data were obtained (typically 20 scans).

For the ESI-MSMS experiments, the ions of interest were mass-selected by the quadrupole mass filter. The precursor ion resolution was adjusted to select only the monoisotopic signal. The selected ions were then submitted to collision against argon in the rf-only hexapole collision cell (pressure estimated at $0.9-1$ mbar). The laboratory frame kinetic energy $\left(\mathrm{E}_{\mathrm{Lab}}\right)$, typically at 45 $\mathrm{eV}$, was selected to afford intense enough fragment ion signals (see presented spectra). All ions coming out of the hexapole cell, either the fragment or the undissociated precursor ions, were finally mass measured with the oa-ToF analyzer. Data were acquired in continuum 
mode until acceptable average data were obtained (typically 20 scans).

\section{Theoretical Calculations}

Since the systems are too large to be fully optimized at a quantum-chemical level, the most stable conformation of each system was first determined using molecular mechanics and molecular dynamics calculations based on the universal force field (UFF) [21, 22] as implemented in the Materials Studio package 4.4 [23]. In both cases, the atomic charges used are those implemented in the COMPASS force field [24]. The cut-off value for the nonbonded interactions has been increased to $30 \AA$ and the conjugated gradient algorithm has been chosen for the geometry optimizations. The most stable conformation for the unfragmented oligomer is obtained by selecting the structure with the lowest energy after optimizing each of the 1000 frames generated every $1 \mathrm{ps}$ from a $1 \mathrm{~ns}$ quenched dynamics $(\mathrm{NVT}, \mathrm{T}=600 \mathrm{~K})$. Starting from this conformation, we used the same procedure to find the most stable conformations of the fragmented systems except that the quenched dynamics are now run at $200 \mathrm{~K}$ since small molecular fragments start leaving the cationized complex at higher temperatures. To analyze the evolution of the system over time and the impact of temperature, molecular dynamics calculations were done at different temperatures within the NVE ensemble. The present analysis will be made from 1000 frames extracted every 10 ps of a $10 \mathrm{~ns}$ dynamics. Note that the temperatures reported hereafter are the temperatures averaged over the $10 \mathrm{~ns}$ molecular dynamics. Since dissociations are kinetic processes, the simulation time of the molecular dynamics is a crucial parameter. Here, we performed 10 ns-long molecular dynamics since the small neutral fragments are leaving, in most cases, the parent species within the first nanoseconds of the dynamics run, as reflected by the limited temperature range where fragmented and nonfragmented systems are coexisting (see the Results section). Finally, density functional theory (DFT) calculations (using the B3LYP functional and a 6-31 $\mathrm{g}(\mathrm{d}, \mathrm{p})$ basis set $[25,26]$ have been performed to evaluate the total energy of the different systems without further geometry optimizations.

\section{Results and Discussion}

In a recent work dealing with the study of metastable dissociations on an orthogonal-axis time-of-flight instrument, we proposed a rapid method for the massscale calibration of the ions generated through the so-called post-pusher decay (PPD) process [27]. The proposed calibrating ion was an ESI-prepared protonated polyhedral oligomeric silsesquioxane (POSS) that reveals multiple and abundant metastable dissociations. The interest of the method was illustrated in the context of the collision-induced dissociation reactions of a sodium-cationized polylactide. The investigated poly- lactide was obtained by an organometallic synthesis using hexadecylamine as the initiator.

As summarized in Scheme 1, when submitted to CID activation, sodium-cationized oligomers of hexadecylamidepolylactide (AlkylNH-PLA-OH$\cdot \mathrm{Na}^{+}$) can suffer from several consecutive and competitive dissociation reactions. First of all, as exemplified in Scheme 1, the losses of 295 and $90 \mathrm{u}$, respectively, routes (a) and (b), can be considered as end-group specific and must arise through Mc-Lafferty-like rearrangements that involve the migration of the hydrogen atom from a pendant methyl group to the oxygen atom of the carbonyl group of the ester moiety. Those results are fully supported by previous papers on the CID dissociations of sodiumcationized PLA oligomers[13, 20]. The involvement of six-membered ring transition states is consistent with the efficiency of such decompositions [14].

The obtained fragment ions can also be involved in further dissociation, as demonstrated by our PPD study [27]. As an example, route (c) was demonstrated to occur in quite good yield, depending on the CID conditions. More interesting because less easy proved was the demonstration that route (d) was really a dissociation pathway for the mass-selected parent ions subjected to collision. Actually, on the basis of the sole analysis of the CID fragments, there is no way to obtain evidences that route (d) is really effective. Indeed, the ions obtained following route (d) exactly possess the same $\mathrm{m} / \mathrm{z}$ ratio than the ions obtained by the consecutive losses of 90 and $72 u$, respectively, route (b) plus route (c). Investigation of metastable ion decompositions was really decisive in solving this problem. A nice feature of the observation of metastable ions in tandem mass spectrometry analysis is that they immediately reveals the identity of their precursors [28]. As a consequence, the observation of metastable ion fragments can help to identify mass transitions in CID spectra. The observation of a metastable transition corresponding to a direct loss of $162 \mathrm{u}$ allowed us to conclude that in CID experiments, sodium-cationized oligomers of PLA undergo consecutive and competitive dissociations [27]. Scheme 1 also reveals the presence of an unexpected $72 \mathrm{u}$ loss directly from the mass-selected parent ions (route (e)). The involved dissociation mechanism requires a complex rearrangement that will be investigated in details in the present paper.

\section{Tandem Mass Spectrometry Results}

In the present study, we will subject to CID analysis several sodium-cationized PLA oligomers. The investigated polymers, presented in Scheme 2, were constructed on the basis of poly(lactic acid) by formally progressively modifying/protecting both end-groups. The exact procedures for the synthesis of those polymers are described in the Experimental section.

When subjected to Electrospray analysis with our QToF 2 instrument, all PLA samples presented in Scheme 2 afford quite abundant signals corresponding 


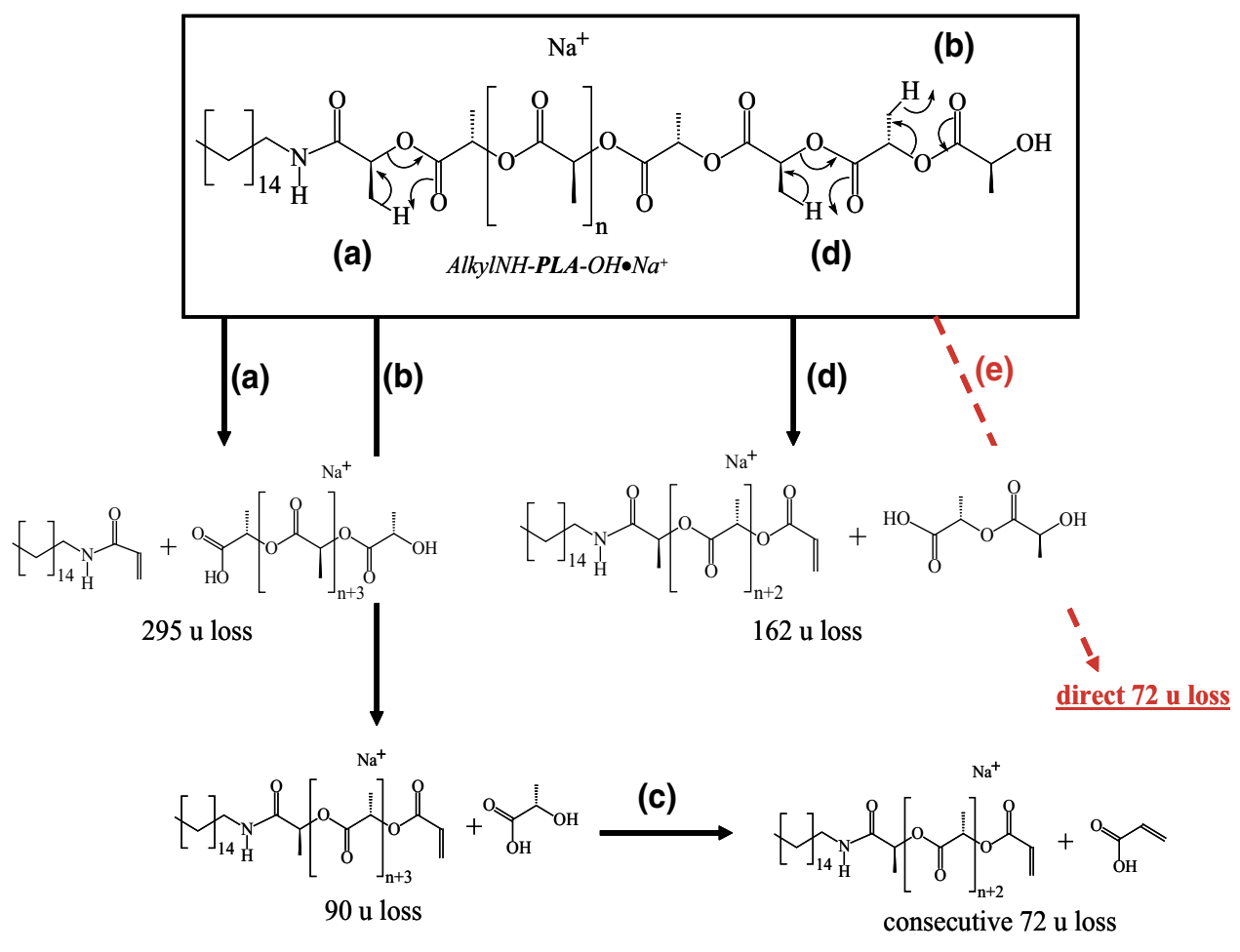

Scheme 1. CID fragmentations of sodium-cationized oligomers of hexadecylamide-polylactide [27].

to the sodium-cationized oligomers. For example, Figure 1 displays the ESI single-stage mass spectrum obtained when analyzing a solution of H-PLA-OH in acetonitrile. In this spectrum, the assignment of the ions constituting the (filled square) ion series to singly charged $\mathrm{H}-\mathrm{PLA}-\mathrm{OH} \cdot \mathrm{Na}^{+}$cations is straightforward, whereas the (filled diamond) ion series is assigned to doubly charged H-PLA-OH-2Na ${ }^{+}$. A third ion series is also detected in the spectrum (for instance, $\mathrm{m} / \mathrm{z} 815$ signal in the inset in Figure 1) whose origin will be described later in the paper.

For the sake of coherence, we will present hereafter the results of the CID experiments realized on oligomer cations containing the same number of repeating units ( $n=11$ in Scheme 2), whatever the end-groups. This will decrease the influence of the molecular mass of the ions on the center-of-mass energy for a given kinetic energy $\left(E_{l a b}\right)$ when comparing the CID spectra of the different oligomer ions.

\section{CID Investigation of $\mathrm{H}-\mathrm{PLA}-\mathrm{OH} \cdot \mathrm{Na}^{+}$Cations}

Figure 2a presents the CID spectrum of the H-PLA$\mathrm{OH} \cdot \mathrm{Na}^{+}$cations $(m / z$ 833, $n=11)$. As expected, collisionexcited $\mathrm{m} / \mathrm{z} 833$ cations competitively expel, in first fragmentation, a molecule of acrylic acid $(72 \mathrm{u})$ and a molecule of lactic acid $(90 \mathrm{u})$ giving rise to fragment cations detected at $m / z 761$ and 743 , respectively. Both processes are likely to involve six-membered ring transition states, as presented in Scheme 3. Both decompositions are end-group specific and could thus be defined as structural indicative. Again, as demonstrated by our recent study [27], all signals observed in Figure 2a can be explained on the basis of competitive and consecutive fragmentations, all of them involving six-membered ring transition states.

Our findings are totally in line with the results from Osaka et al. for CID experiments on $\mathrm{Na}^{+}$-cationized linear PLA [20]. In particular, they argued that three dissocia-

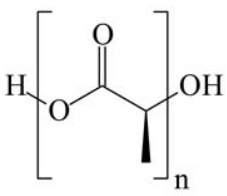

H-PLA-OH<smiles>C=C(O)C(=O)OCC</smiles>

$\mathrm{CH}_{3}-\mathrm{PLA}-\mathrm{OH}$<smiles>CCOC(=O)C(C)OC(C)=O</smiles>

$\mathrm{CH}_{3}-\mathrm{PLA}-\mathrm{O}(\mathrm{C}=\mathrm{O}) \mathrm{CH}_{3}$

Scheme 2. PLA oligomers selected for the mechanistic experimental and theoretical study. 


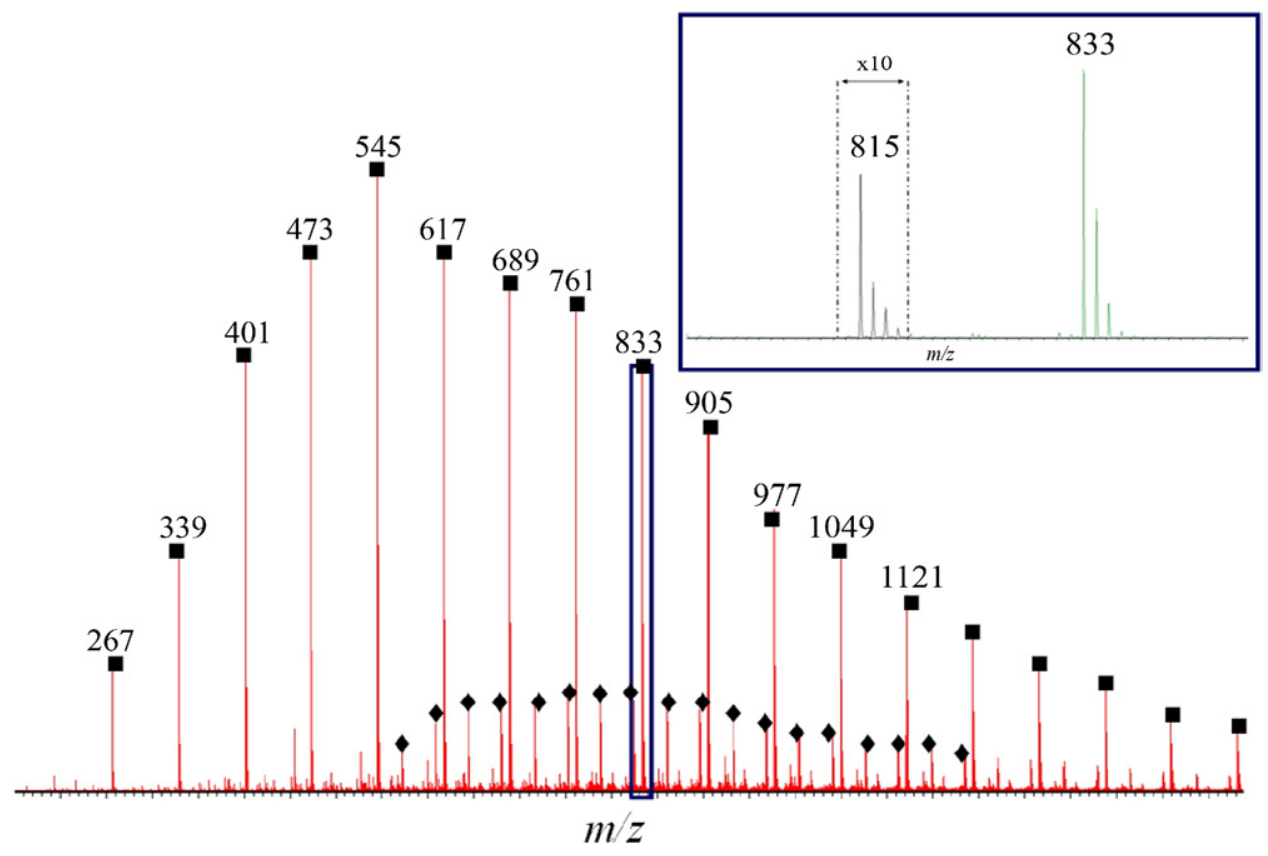

Figure 1. Single Stage ESI-ToF mass spectrum of polylactic acid. Inset corresponds to an expanded view between $\mathrm{m} / \mathrm{z} 800$ and 850 .

tions pathways occur to account for the production of all CID fragment ions. In that paper, they proposed that fragment ions formed by loss of neutral molecules from

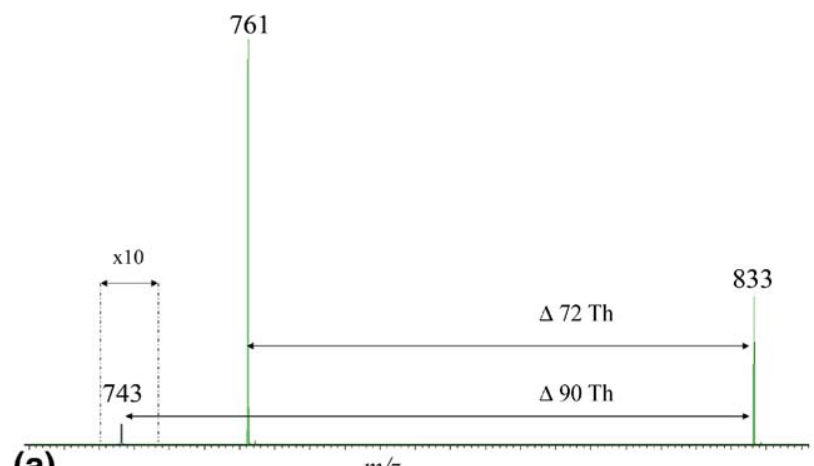

(a) $m / 2$

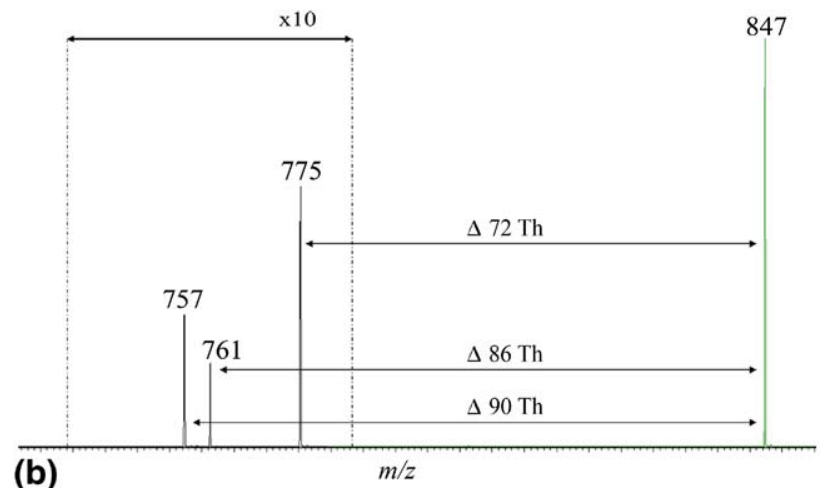

Figure 2. (a) CID spectrum of $\mathrm{Na}^{+}$cationized H-PLA-OH $(\mathrm{m} / \mathrm{z}$ 833; argon collision gas; $45 \mathrm{eV}$ collision energy). (b) CID spectrum of $\mathrm{Na}^{+}$cationized $\mathrm{CH}_{3}-\mathrm{PLA}-\mathrm{OH}(\mathrm{m} / \mathrm{z}$ 847; argon collision gas; 45 $\mathrm{eV}$ collision energy). the $\alpha$ and $\omega$ positions constitute the so-called $\mathrm{A}$ and $\mathrm{B}$ ion series, see Scheme 3. In our case, ion series $A$ is initiated by a $72 \mathrm{u}$ loss, whereas ion series B starts with the loss of $90 \mathrm{u}$. They also pointed out the presence of a third dissociation route, ion series $C$, which would correspond to the elimination of the monomeric lactic acid unit (72 u loss) involving the rearrangement of a hydrogen atom. Starting from $\mathrm{H}-\mathrm{PLA}-\mathrm{OH} \cdot \mathrm{Na}^{+}$cations, ions generated following pathway $\mathrm{C}$ (72 u loss) cannot be unambiguously identified since those fragment ions coincide with those of the ion series A. They obtained some evidences for the occurrence of this third dissociation route when investigating the CID behavior of the solvolysis products obtained by degradation in methanol of linear poly(lactic acid). Indeed, in the CID spectrum of their $\left[\mathrm{M}_{n}+\mathrm{MeOH}+\mathrm{Na}^{+}\right]$cations $(\mathrm{M}=$ lactic acid monomer unit), they observed three types of product ions, i.e., the $\mathrm{A}$ and $\mathrm{B}$ series together with the distinct $\mathrm{C}$ ion series [20]. Their main conclusion was that the production of $\mathrm{C}$ fragment ions probably involves a rearrangement of the hydrogen atom at the $\omega$ end-group [20].

To shed further light on this intriguing dissociation pathway, we decided to study in more details the CID behavior of end-group modified polylactides. For the sake of direct comparison with the results of Osaka et al., we built a polymer whose $\alpha$ end-group was modified by the formal replacement of the $\mathrm{HO}$ - group by a $\mathrm{CH}_{3} \mathrm{O}-$ moiety, namely the $\alpha$-methyl, $\omega$-hydroxylpolylactide $\left(\mathrm{CH}_{3}\right.$-PLA-OH).

\section{$\mathrm{CID}$ Investigation of $\mathrm{CH}_{3}-\mathrm{PLA}-\mathrm{OH} \cdot \mathrm{Na}^{+}$Cations}

When submitted to collision-induced dissociation against argon in the hexapole cell of our QToF2 instrument, 


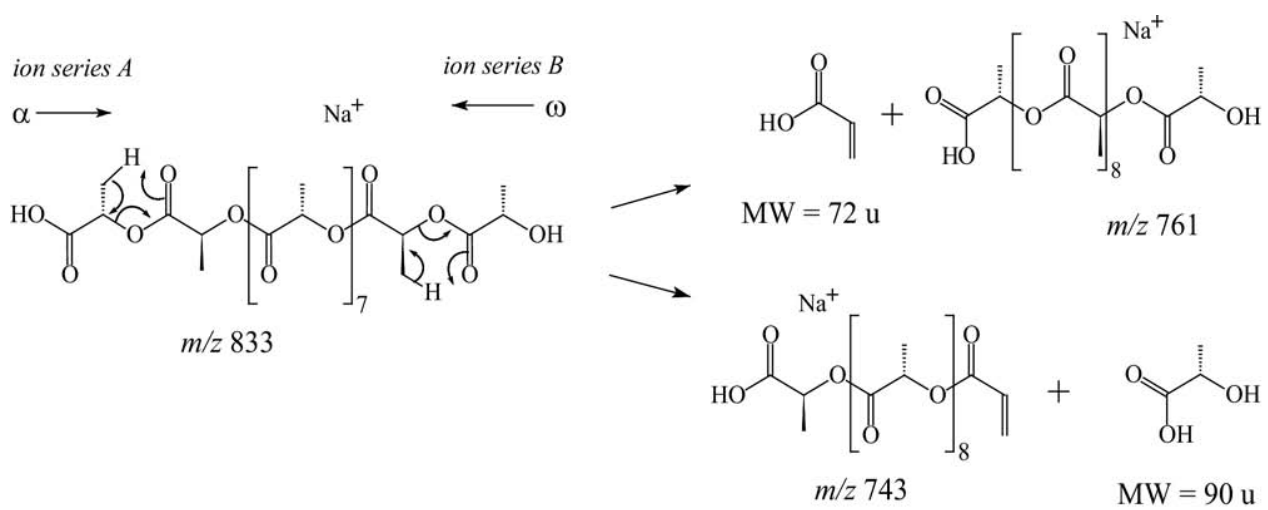

Scheme 3. Collision-induced dissociation of $\mathrm{Na}^{+}$-cationized H-PLA-OH oligomers: end-group specific competitive decompositions.

mass-selected $\mathrm{m} / \mathrm{z} 847$ cations, corresponding to the $\mathrm{CH}_{3}$ PLA-OH $\cdot \mathrm{Na}^{+}$cations containing 11 lactic acid monomer units, exhibit three competitive eliminations. As exemplified in Figure $2 \mathbf{b}$, collisionally-excited $\mathrm{m} / \mathrm{z} 847$ cations competitively dissociate affording $\mathrm{m} / \mathrm{z} 775,761$, and 757 ions that, respectively, originate from 72,86 , and $90 \mathrm{u}$ losses. Based on Osaka's nomenclature [20], those ions belong to the $\mathrm{C}, \mathrm{A}$, and $\mathrm{B}$ ion series, respectively (Scheme S.I.1, which can be found in the electronic version of this article). Obviously, the loss of acrylic acid $(72 \mathrm{u})$ that was previously identified as an endgroup specific decomposition for H-PLA-OH $\cdot \mathrm{Na}^{+}$cations (ion series A in Scheme 3) is replaced by a loss of methyl acrylate (86 u loss, see Scheme S.I.1 for details). Together with the $90 \mathrm{u}$ loss (lactic acid loss - ion series B in Scheme 3), the decomposition leading to the methyl acrylate elimination can also be considered as structural characteristic (see Scheme S.I.1). As far as ion series $C$ is concerned (72 u loss), it was proposed by Osaka et al. that the production of the $C$ fragment ions involves a rearrangement of the hydrogen atom at the $\omega$ end-group [20].

To further assess this hypothesis, we chemically modified our $\mathrm{CH}_{3}$-PLA-OH oligomers by reaction with acetyl chloride to obtain a new set of oligomers whose $\omega$ end-group now presents an ester function. The $\mathrm{CH}_{3}-$ PLA-O $(\mathrm{C}=\mathrm{O}) \mathrm{CH}_{3}$ polymer is then submitted to Electrospray Ionization and the CID spectrum of the $\mathrm{CH}_{3}-$ PLA-O $(\mathrm{C}=\mathrm{O}) \mathrm{CH}_{3} \cdot \mathrm{Na}^{+}$cations is then recorded.

\section{CID Investigation of $\mathrm{CH}_{3}-\mathrm{PLA}-\mathrm{O}(\mathrm{C}=\mathrm{O}) \mathrm{CH}_{3} \cdot \mathrm{Na}^{+}$} Cations

Figure 3 presents the CID spectrum of the $\mathrm{CH}_{3}$-PLA$\mathrm{O}(\mathrm{C}=\mathrm{O}) \mathrm{CH}_{3} \cdot \mathrm{Na}^{+}$cations possessing 11 lactic acid monomeric units. The analysis of this spectrum reveals that, when subjected to collision, gas-phase $\mathrm{m} / \mathrm{z} 889$ cations competitively yield $m / z 829,817,803$, and 757 cations as fragment ions. The dissociation reactions involved in these mass transitions are respectively the losses of acetic acid (60 u loss), $\left[\mathrm{C}_{3} \mathrm{H}_{4} \mathrm{O}_{2}\right]$ (72 u loss), $\left[\mathrm{C}_{4} \mathrm{H}_{6} \mathrm{O}_{2}\right]$ (86 u loss), and $\left[\mathrm{C}_{5} \mathrm{H}_{8} \mathrm{O}_{4}\right]$ (132 u loss). The main piece of information obtained from this CID spectrum is that, whatever the nature of the end-groups either at the $\alpha$ or $\omega$ position, the loss of $72 \mathrm{u}$ is always detected and cannot be envisaged as a structural characteristic dissociation. Before entering into more details for this peculiar reaction, it is interesting to analyze the other competitive fragmentations leading, respectively, to the losses of 60, 86, and $132 \mathrm{u}$. The loss of acetic acid (60 u) from the acetic acid ester end-group was definitively expected and is likely to also involve a six-membered ring transition-state (see Scheme S.I.2). Fragment ions related to the end-group specific decompositions, namely ion series A and B, are also detected and the pathways leading to their observation are similar to the end-group decomposition reactions presented in Scheme 3.

As far as the $72 \mathrm{u}$ loss is concerned, we have now strong evidences that this peculiar reaction cannot be related to an ordinary elimination process occurring from the $\alpha$ or $\omega$ position of the polymer chain (ion series A and B). Such a process is definitively not structural indicative and deserves to be examined carefully.

\section{CID Investigation of Cyclic-PLA $\cdot \mathrm{Na}^{+}$Cations}

To shed light into the $72 \mathrm{u}$ loss, the CID behavior of sodium-cationized cyclic polylactide oligomers is worth

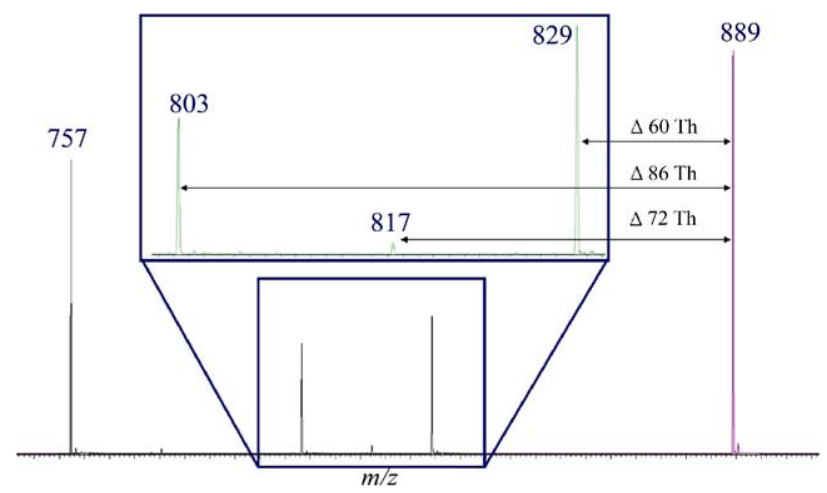

Figure 3. $\mathrm{CID}$ spectrum of $\mathrm{Na}^{+}$cationized $\mathrm{CH}_{3}-\mathrm{PLA}-\mathrm{O}(\mathrm{C}=$ O) $\mathrm{CH}_{3}(\mathrm{~m} / \mathrm{z}$ 889; argon collision gas; $45 \mathrm{eV}$ collision energy). 
to be analyzed. Indeed, as reported by Osaka et al. [20] upon collisional activation of mass-selected cyclicPLA $\cdot \mathrm{Na}^{+}$ions, fragment ions resulting from sequential elimination of the monomeric residue $(72 \mathrm{u})$ are detected, although no mechanistic description was given. Nevertheless, we suspect that similarities can be expected between the CID reactions of cyclic-PLA $\cdot \mathrm{Na}^{+}$ ions and the reaction leading to the $72 \mathrm{u}$ loss in the linear chain.

In Figure 1, the third ion series could in principle correspond to cyclic-PLA $\cdot \mathrm{Na}^{+}$ions, since the measured mass-to-charge ratios are multiple of $72 \mathrm{u}$ after having subtracted the mass of the sodium ion. However, isomeric species such as a linear PLA $\cdot \mathrm{Na}^{+}$ion presenting, as end-groups, a carboxylic acid and an acrylic acid ester functions (Scheme S.I.3) can also be envisaged. Therefore we have first to unambiguously determine the cyclic nature of the detected species. The linear $\mathrm{PLA} \cdot \mathrm{Na}^{+}$ions could be for instance prepared in the gas-phase by in-source CID of H-PLA-OH- $\mathrm{Na}^{+}$cations, see Scheme 3. Clear evidences for the cyclic structure in the first sample came from an elegant H/D exchange experiment in solution before the ESI process (see Figure S.I.1 for more details).

Upon collisional activation (see Figure S.I.2), $m / z 815$ cations, corresponding to cyclic-PLA $\cdot \mathrm{Na}^{+}$ions, are decomposed by consecutive losses of $72 \mathrm{u}$. Competitive reactions are also probably responsible for the detected CID fragments. As for a mechanistic explanation, we could propose that collisionally-excited cations first undergo a ring-opening reaction as presented in Scheme 4.

This backbone cleavage is likely to involve a sixmembered ring transition-state and will afford a new sodium-cationized polylactide whose end-groups are readily identified as a carboxylic acid group and an acrylic acid ester function at the $\alpha$ and $\omega$ positions, respectively, see Scheme 4 . The obtained cations can now expel neutral acrylic acid following a consecutive dissociation reaction. Interestingly, this initial $72 \mathrm{u}$ loss gives rise to $\mathrm{Na}^{+}$-cationized PLA possessing acrylic acid as the $\omega$ end-group. In other words, the obtained $m / z 743$ cations can suffer from exactly the same dissociation process.

Having demonstrated that the sodium-cationized cyclic-PLA oligomers can suffer from a backbone cleavage before decomposition, we propose that such a reaction is also occurring for any sodium-cationized polylactide oligomers. When starting from linear PLA cations, this peculiar backbone cleavage process will afford sodium-bound dimer cationic species. Such species are stabilized by the electrostatic association of two linear PLA oligomers with a central sodium cation (Scheme S.I.4). The interesting feature of this hypothesis is that, irrespective of the nature of the end-groups, this backbone cleavage will invariably lead to the production of two new end-groups that are a carboxylic acid group and an acrylic acid ester function (see Scheme 4 in the analogous case of the cyclic species). This new cationic species is then prone to decomposition by loss of acrylic acid and this $72 \mathrm{u}$ elimination is definitively not structural indicative.

In the following part of this work, we will present the results of theoretical calculations on the stability of the species obtained by the collision-induced dissociation of $\mathrm{CH}_{3}-\mathrm{PLA}-\mathrm{O}(\mathrm{C}=\mathrm{O}) \mathrm{CH}_{3} \cdot \mathrm{Na}^{+}$cations containing 11 lactic acid monomer units.
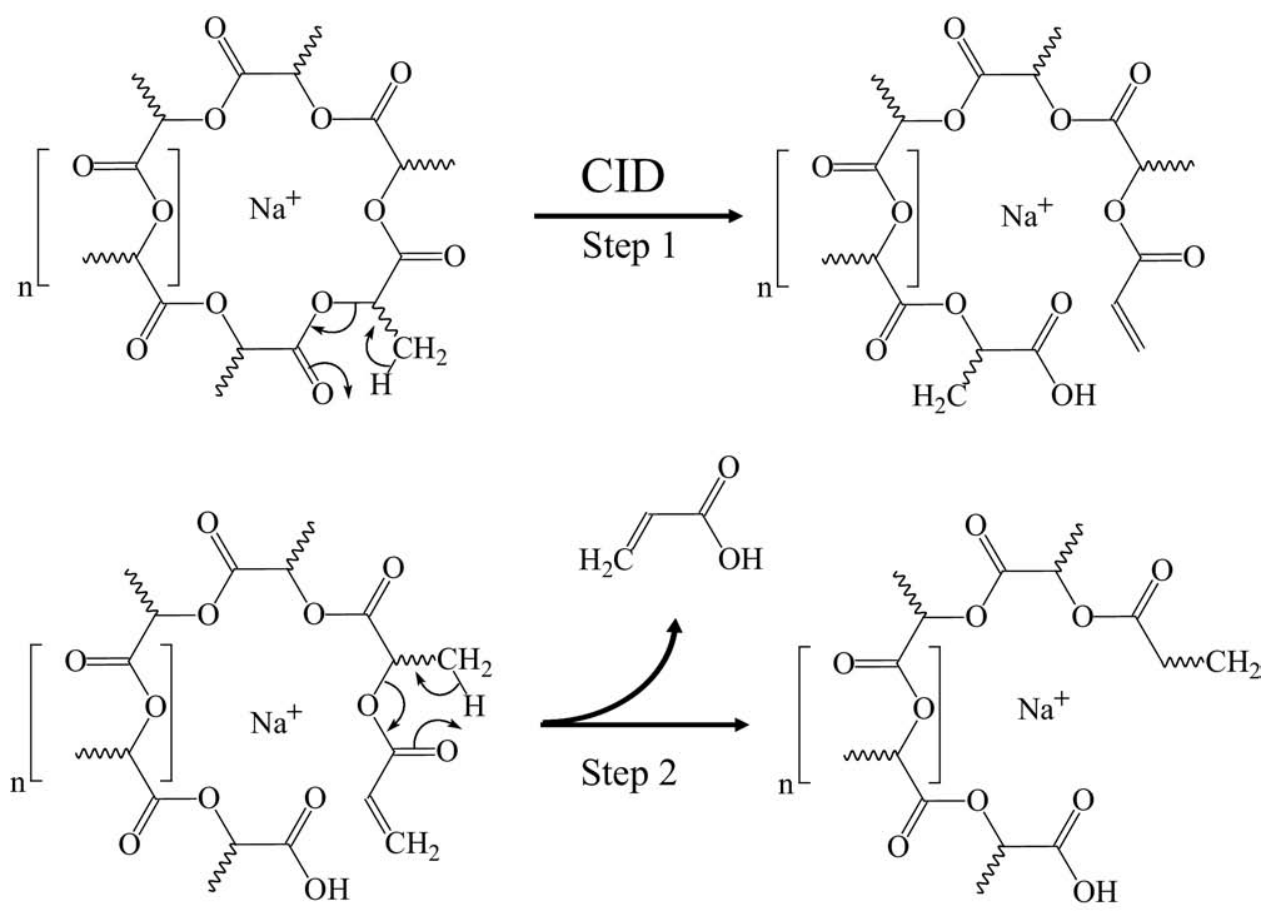

Scheme 4. CID investigation of cyclic PLA $\cdot \mathrm{Na}^{+}$cations: origin of the $72 \mathrm{u}$ loss. 


\section{Theoretical Calculations}

Since a $72 \mathrm{u}$ elimination is observed, this implies that multiple rearrangement processes occur in the cationized oligomer and that the stability of the fragmented systems is not too different from that of the complex after the initial rearrangement. This has been verified at the DFT level by calculating the total energy of different fragmented systems derived from a $\mathrm{CH}_{3}-\mathrm{PLA}-\mathrm{O}(\mathrm{C}=$ O) $\mathrm{CH}_{3} \cdot \mathrm{Na}^{+}$cation containing 11 lactic acid monomer units. As stated in the Experimental section, no geometry optimizations were performed at the DFT level. Instead, the calculations were performed on the most stable structures determined from the Molecular Mechanics approach. The systems presented in Figure 4 are schematic representations of sodium-cationized PLA that have undergone (a) backbone cleavage(s), without any loss of neutral molecules. In other words, these structures correspond to complex cations in which different neutral moieties are associated through electrostatic interactions with the sodium ion. Figure 4 also displays the three-dimensional structures of the most stable conformer of systems 1 and 5 . In both structures, a significant number of oxygen atoms are pointing towards the cationic sodium atom to enhance the electrostatic interactions. Figure 5 shows that the energy differences between the systems under study is small enough $(<1 \mathrm{eV})$ to envision a possible $72 \mathrm{u}$ elimination in the center of the oligomer upon collision. As a matter of fact, system $\mathbf{5}$ is more stable than systems $\mathbf{2}$ or $\mathbf{3}$ for whose end-group elimination is unambiguously evidenced experimentally. Note that system 4 (a precursor of system 5) also exhibits a lower energy than systems 2 and 3. This increased stability for systems 4 and 5 versus systems 2 and 3 can be explained by the fact that a backbone cleavage at the center of the oligomer allows for more interactions between the negatively

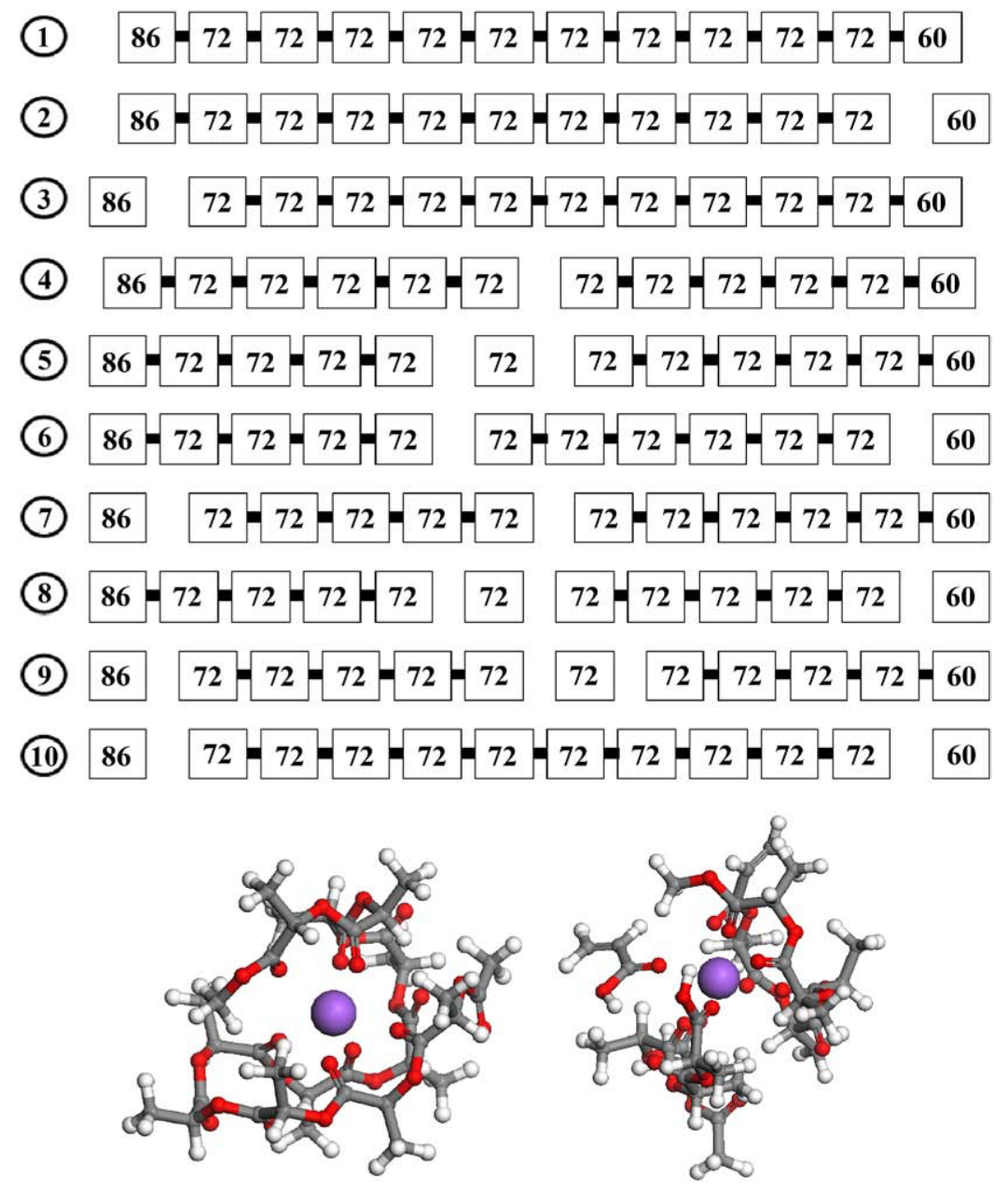

Figure 4. (up) Schematic representation of the different systems considered before any loss of neutral fragments. For the sake of clarity, the sodium cation has been omitted. The systems are represented as a linear array, while the structures optimized at the molecular mechanics level are folded around the sodium cation; (bottom) 3-D representations of the most stable conformation of systems $\mathbf{1}$ (left) and 5 (right); carbon, oxygen, hydrogen, and sodium atoms are displayed in grey, red, blank, and purple, respectively. For system 5 , the $72 \mathrm{u}$ fragment (middle left) resulting from two central hydrogen shifts can be now easily expelled. 


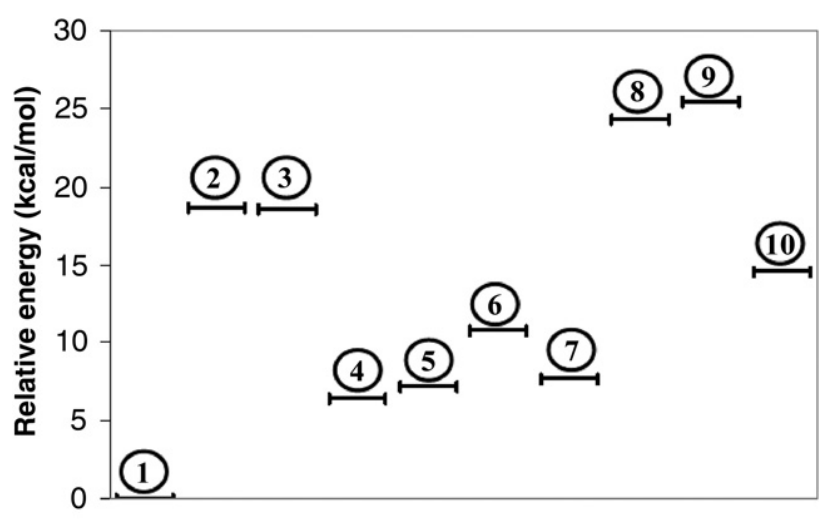

System numbers

Figure 5. Relative energies of the different systems represented at Figure 4 as calculated at the DFT/B3LYP level from the most stable conformation provided by Molecular Mechanics.

charged oxygen atoms and the positively charged sodium atom.

Since system 5 clearly co-exists with other fragmented systems, the appearance of the peak at $\mathrm{m} / \mathrm{z} 817$ in Figure 3 also implies that the energy needed to promote this $72 \mathrm{u}$ fragment far away from the system should not exceed by far the energy needed to eliminate the end-groups. This has been assessed by running several $10 \mathrm{~ns}$ molecular dynamics simulations (in the NVE ensemble) on system $\mathbf{5}$ at different temperatures to evaluate the temperature at which the central $72 \mathrm{u}$ fragment leaves the cationic species. Note that the temperature in molecular dynamics is directly linked to the kinetic energy provided to the system (this transfer is done experimentally by the collisions within the mass spectrometer) and can therefore be viewed as a parameter reflecting the amplitude of the binding energy between the fragment and the remaining cationic species. Since the formation of system 5 requires two rearrangement processes, we did similar $10 \mathrm{~ns}$ molecular dynamics simulations on systems 6 and 7 which also result from two rearrangements, i.e., one in the middle of the oligomer and the other at one end. A schematic view of the molecular dynamics results is presented in Figure 6. It shows that the $60 \mathrm{u}$ fragment starts leaving the cationic species (from system 6) at around 240-250 $\mathrm{K}$ while the $72 \mathrm{u}$ (from system 5) and $86 \mathrm{u}$ (from system 7) fragments are expelled at higher temperature (between 270 and $280 \mathrm{~K}$ ). Note that the kinetic energy corresponding to a temperature difference of $30 \mathrm{~K}$ is only $10 \mathrm{kcal} / \mathrm{mol}$. These results thus point to competitive elimination processes for the three fragments and confirm that the peak at $\mathrm{m} / \mathrm{z} 817$ (loss of $72 \mathrm{u}$ ) can originate from one of the central monomeric units.

While the intensity of the peaks cannot be directly related to the ease of elimination of the fragments since successive eliminations occur, we also ran similar molecular dynamics simulations on systems $\mathbf{8 , 9}$, and $\mathbf{1 0}$ where two fragments are prepared to be expelled from the complex ion. This allows us to evaluate the relative ease of elimination of each fragment (system 8 - loss of 60 and $72 \mathrm{u}$; system 9 - loss of 72 and $86 u$; system 10 - loss of 60 and $86 \mathrm{u}$ ). Our simulations clearly show that the elimination of the $60 \mathrm{u}$ fragment is much easier compared to the others (from systems 8 and 10), whereas system 9 points to a competitive elimination of the 72 and $86 \mathrm{u}$ fragments even though the $72 \mathrm{u}$ elimination can be sometimes observed at lower temperature.

The theoretical results are thus in full consistency with the experimental spectrum (Figure 3). The intense peak at $m / z 829$ reflects the easier departure of the $60 \mathrm{u}$ end-group. However, while the simulations point to competitive eliminations for the 72 and $86 \mathrm{u}$ fragments from system 9 , the peak at $m / z 803$ ( $86 \mathrm{u}$ loss) is more intense than that at $\mathrm{m} / \mathrm{z} 817$ (72 $\mathrm{u}$ loss). As an explanation, the departure of the $86 \mathrm{u}$ end-group may also only require one cleavage and could then be envisaged from system 3 in Figure 4. Since the $60 \mathrm{u}$ end-group leaves the complex very easily and since the corresponding peak at $m / z 829$ is not much more intense than the peak at $m / z 803$ (86 u loss), we also conclude that successive eliminations of $72 \mathrm{u}$ fragments also occur quickly from the $\mathrm{m} / \mathrm{z} 829$ complex leading to the $\mathrm{m} / \mathrm{z} 757$ intense signal (see Figure 3).

\section{Conclusions}

The low-kinetic energy collision-induced dissociation (CID) behavior of different sodium-cationized polylactide (PLA) oligomers was thoroughly investigated to shed some light on the analytical potentialities of CID experiments in the context of polymer characterization. Investigations of several end-groups modified PLA reveal that, upon collisional activation, PLA. $\mathrm{Na}^{+}$systematically suffers from end-group specific dissociations. Those dissociations proceed through favorable six-membered ring transition states (McLafferty-like rearrangement). Consecutive and competitive fragmentations are also highlighted and were shown to corre-

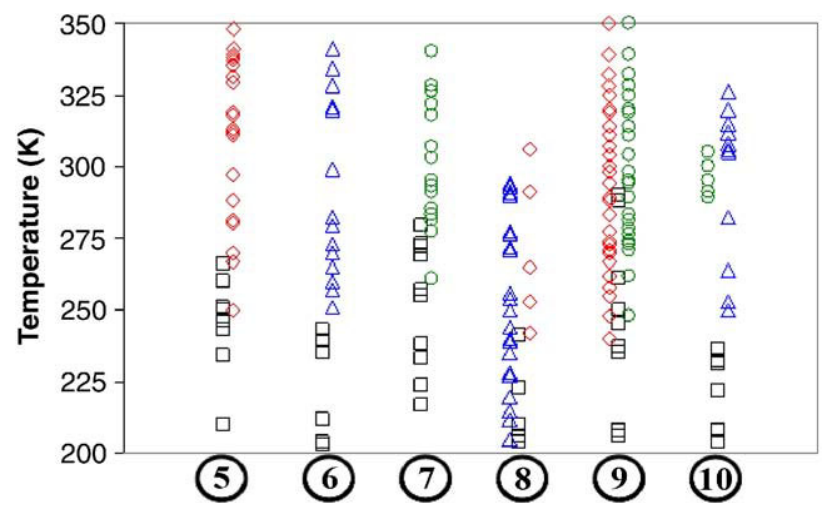

Figure 6. Schematic representation of the departure temperatures of the different fragments (black squares: no departure; red diamonds: departure of the $72 \mathrm{u}$ fragment; blue triangles: departure of the $60 \mathrm{u}$ fragment; and green circles: departure of the $86 \mathrm{u}$ fragment) for systems 5 to $\mathbf{1 0}$. 
spond to progressive fragmentations of the oligomer chain starting from both the end-groups. In addition to those structurally-indicative dissociations, we demonstrated that collisionally-excited PLA.Na ${ }^{+}$competitively suffer from two consecutive backbone cleavages leading to sodium-bound dimer and trimer cations that ultimately lead to the loss of a monomeric residue that corresponds to neutral acrylic acid. The experimental observations, performed on a hybrid Q-ToF instrument, were totally corroborated by a theoretical study involving DFT calculations, molecular mechanics and molecular dynamics simulations.

\section{Acknowledgments}

The MS laboratory acknowledges the Fonds de la Recherche Scientifique (FRS-FNRS) for its contribution to the acquisition of the QToF2 Mass Spectrometer. J.C., P.G., O.C., and J.D.W. are FNRS research fellows. The authors acknowledge that this work was partially supported by the Belgian Federal Government Office of Science Policy (SSTC-PAI 6/27).

\section{Appendix A Supplementary Material}

Supplementary material associated with this article may be found in the online version at doi:10.1016/ j.jasms.2010.03.026.

\section{References}

1. Liem, M. D.; Zegel, H. G.; Balduini, F. C.; Turner, M. L.; Becker, J. M.; Caballerosaez, A. Repair of Achilles Tendon Ruptures with a Polylactic Acid Implant: Assessment with MR Imaging. Am. J. Roentgenol. 1991, 4, 769-773.

2. Shikinami, Y.; Matsusue, Y.; Nakamura, T. The Complete Process of Bioresorption and Bone Replacement Using Devices Made of Forged Composites of Raw Hydroxyapatite Particles/Poly L-Lactide (F-u-HA/ PLLA). Biomaterials 2005, 26, 5542-5551.

3. Boccaccini, A. R.; Blaker, J. J.; Maquet, V.; Day; R. M.; Jérôme, R. Preparation and Characterization of Poly(Lactide-co-Glycolide) (PLGA) and PLGA/Bioglass Composite Tubular Foam Scaffolds for Tissue Engineering Applications. Mat. Sci. Eng. C-Bio. S 2005, 1, 23-31.

4. Zhang, L.; Nederberg, F.; Messman, J. M.; Pratt, R. C.; Hedrick, J. H.; Wade, C. G. Organocatalytic Stereoselective Ring-Opening Polymerization of Lactide with Dimeric Phosphazene Bases. J. Am. Chem. Soc. 2007, $129,12610-12611$.

5. Kamber, N. E.; Jeong, W.; Waymouth, R. M.; Pratt, R. C.; Lohmeijer, B. G. G.; Hedrick, J. L. Organocatalytic Ring-Opening Polymerization. Chem. Rev. 2007, 107, 5813-5840.

6. Coulembier, O.; Sanders, D. P.; Nelson, A.; Hollenbeck, A. N.; Horn, H. W.; Rice, J. E.; Fujiwara, M.; Dubois, P.; Hedrick, J. L. HydrogenBonding Catalysts Based on Fluorinated Alcohol Derivatives for Living Polymerization. Angew. Chem. 2009, 121, 5272-5275.
7. Coulembier, O.; Kiesewetter, M. K.; Mason, A.; Dubois, P.; Hedrick, J. L.; Waymouth, R. M. A Distinctive Organocatalytic Approach to Complex Macromolecular Architectures. Angew. Chem. Int. Edit. 2007, $46,1-4$

8. Montaudo, G.; Lattimer, R. P. Mass Spectrometry of Polymers; CRC Press: Boca Raton, 2002; pp 419-523.

9. Nielen, M. W. F. MALDI Time-of-Flight Mass Spectrometry of Synthetic Polymers. Mass Spectrom. Rev. 1999, 18, 309-344.

10. Arnould, M. A.; Wesdemiotis, C.; Geiger, R. J.; Park, M. A.; Buehner R. W.; Vandervorst, D. Structural Characterization of Polyester Copolymers by MALDI Mass Spectrometry. Prog. Org. Coat. 2002, 45, 305-312.

11. Chaicharoen, K.; Polce, M. J.; Singh, A.; Pugh, C.; Wesdemiotis, C. Characterization of Linear and Branched Polyacrylates by Tandem Mass Spectrometry. Anal. Bioanal. Chem. 2008, 392, 595-607.

12. Arnould, M. A.; Vargas, R.; Buehner, R. W.; Wesdemiotis, C. Tandem Mass Spectrometry Characteristics of Polyester Anions and Cations Formed by Electrospray Ionization. Eur. J. Mass Spectrom. 2005, 11, 243-256.

13. Adamus, G. Structural Analysis of Poly[(R,S)-3-Hydroxybutyrate-co-LLactide] Copolyesters by Electrospray Ionization Ion Trap Mass Spectrometry. Rapid Commun. Mass Spectrom. 2007, 21, 2477-2490.

14. Sleno, L.; Volmer, D. A. Ion Activation Methods for Tandem Mass Spectrometry. J. Mass Spectrom. 2004, 39, 1091-1112.

15. Jackson, A. T.; Bunn, A.; Chisholm, M. S. Utilizing Matrix-Assisted Laser Desorption/Ionization Techniques for the Generation of Structural Information from Different End-Group Functionalized Poly(Methyl Methacrylate)s. Polymer 2008, 49, 5254-5261.

16. Wollyung, K. M.; Wesdemiotis, C.; Kennedy, J. P. Synthesis and Mass Spectrometry Characterization of Centrally and Terminally AmineFunctionalized Polyisobutylenes. J. Polym. Sci. Part A: Polym. Chem. 2004, 43, 946-958.

17. Polce, M. J.; Ocampo, M.; Quirk, R. P.; Wesdemiotis, C. Tandem Mass Spectrometry Characteristics of Silver-Cationized Polystyrenes: Backbone Degradation Via Free Radical Chemistry. Anal. Chem. 2008, 2, 347-354.

18. Jackson, A. T.; Yates, H. T.; Scrivens, J. H.; Green, M. R.; Bateman, R. H Using Matrix Assisted Laser Desorption/Ionization-Collision Induced Dissociation for the Generation of Structural Information from Poly(Alkyl Methacrylate)s. J. Am. Soc. Mass Spectrom. 1997, 8, 1206-1213.

19. Jackson A. T.; Green, M. R.; Bateman, R. H. Generation and End-Group Information from Polyethers by Matrix-Assisted Laser Desorption/ Ionization Collision-Induced Dissociation Mass Spectrometry. Rapid Commun. Mass Spectrom. 2006, 20, 3542-3550.

20. Osaka, I.; Watanabe, M.; Takama, M.; Murakami, M.; Arakawa, R. Characterization of Linear and Cyclic Polylactic Acids and Thei Solvolysis Products by Electrospray Ionization Mass Spectrometry. J. Mass Spectrom. 2006, 41, 1369-1377.

21. Casewit, C. J.; Colwell, K. S.; Rappé, A. K. Application of a Universal Force-Field to Organic-Molecules. J. Am. Chem. Soc. 1992, 114, 1003510046.

22. Rappé, A. K.; Casewit, C. J.; Colwell, K. S.; Goddard, W. A.; Skiff, W. M. UFF. A Full Periodic-Table Force-Field for Molecular Mechanics and Molecular-Dynamics Simulations. J. Am. Chem. Soc. 1992, 114, $10024-$ 10035.

23. MS Modeling Ver. 4.4.0.0, Accelrys Software Inc: San Diego 2008.

24. Sun, H. COMPASS: An ab Initio Force-Field Optimized for CondensedPhase Applications-Overview with Details on Alkane and Benzene Compounds. J. Phys. Chem. B 1998, 102, 7338-7364.

25. Becke, A. D. Density-Functional Thermochemistry. 3. The Role of Exact Exchange. J. Chem. Phys. 1993, 98, 5648-5652.

26. Lee, C.; Yang, W.; Parr, R. G. Development of the Colle-Salvetti Correlation-Energy Formula into a Functional of the Electron Density. Phys. Rev. B 1988, 37, 785-789.

27. De Winter, J.; Goffin, A. L. Coulembier, O ; Dubois, P.; Flammang, R. Gerbaux, P. Metastable Processes Investigated on an Orthogonal-Axis Time-of-Flight Instrument: Mass-Scale Calibration and Application. Eur. J. Mass Spectrom. 2009, 15, 431-437.

28. Cooks, R. G.; Beynon, J. H.; Caprioli, R. M.; Lester, G. R. Metastable Ion Elsevier: Amsterdam, 1973; pp 89-153. 